\title{
Development and validation of a multi-parameter nomogram for predicting prostate cancer: A retrospective analysis from Handan Central Hospital in China
}

\author{
Libin Nan ${ }^{1}$, Kai Guo ${ }^{2}$, Mingmin $\mathrm{Li}^{3}{ }^{3}, \mathbf{Q i} \mathbf{W u}^{1}$, Shaojun Huo \\ ${ }^{1}$ Department of Urology, Handan Central Hospital, Handan, Hebei, China \\ 2 Cardiac Department, Turku City Hospital, Turku, Varsinais-suomi, Finland \\ 3 Out-patient department, Handan Central Hospital, Handan, Hebei, China \\ Corresponding Author: Libin Nan \\ Email address: nanlibin@126.com
}

Background To explore the possible predicting factors related to prostate cancer and develop a validated nomogram for predicting the probability of patients with prostate cancer. Method Clinical data of 697 patients who underwent prostate biopsy in Handan Central Hospital from January 2014 to January 2020 were retrospectively collected. Cases are randomized into two groups: $80 \%$ (548 cases) as the development group, and $20 \%$ (149 cases) as the validation group. Univariate and multivariate logistic regression analyses were performed to determine the independent risk factors for prostate cancer. The nomogram prediction model was generated using the finalized independent risk factors. Decision curve analysis (DCA) and the area under receiver operating characteristics curve (ROC) of both development group and validation group were calculated and compared to validate the accuracy and efficiency of the nomogram prediction model. Clinical utility curve (CUC) helped to decide the desired cut-off value for the prediction model. Comparison of the established nomogram with Prostate Cancer Prevention Trial Derived Cancer Risk Calculator (PCPT-CRC) and other domestic prediction models using the entire study population is conducted. Results The independent risk factors determined through univariate and multivariate logistic regression analyses were: age, TPSA, fPSA, PV, DRE, TRUS and BMI. Nomogram prediction model was developed with the cut-off value of 0.31 . The AUC of development group and validation group were 0.856 and 0.797 respectively. DCA exhibits consistent observations with the findings. Through validating our prediction model as well as other three domestic prediction models based on the entire study population of 697 cases, our prediction model demonstrated significantly higher predictive value than all the other models. Conclusion The nomogram for predicting prostate cancer can facilitate more accurate evaluation of the probability of having prostate cancer, and provide better ground for prostate biopsy. 
1 Development and validation of a multi-parameter nomogram for

2 predicting prostate cancer: A retrospective analysis from Handan

3 Central Hospital in China

4 Libin $\mathrm{Nan}^{1}, \mathrm{Kai} \mathrm{Guo}^{2}$, Mingmin $\mathrm{Li}^{3}$, Qi Wu${ }^{4}$, Shaojun Huo

$5{ }^{1}$ Department of Urology, Handan Central Hospital, Handan, Hebei, China 056001

$6 \quad{ }^{2}$ Cardiac Department, Turku City Hospital, Turku, Finland 20701

$7 \quad{ }^{3}$ Out-patient department, Handan Central Hospital, China, Handan 056001

$8{ }^{4}$ Department of Urology, Handan Central Hospital, Handan, Hebei, China 056001

$9 \quad{ }^{5}$ Department of Urology, Handan Central Hospital, Handan, Hebei, China 056001

Corresponding Author:

Name: Shaojun Huo

Address: Zhonghua street 15, Hanshan district, Handan, Hebei, China

Post code: 056001

E-mail: hdmnwk@sina.com

Tel: (0086) 0310-2118083 


\section{Abstract}

Background To explore the possible predicting factors related to prostate cancer and develop a validated nomogram for predicting the probability of patients with prostate cancer.

Method Clinical data of 697 patients who underwent prostate biopsy in Handan Central Hospital from January 2014 to January 2020 were retrospectively collected. Cases are randomized into two groups: $80 \%$ (548 cases) as the development group, and 20\% (149 cases) as the validation group. Univariate and multivariate logistic regression analyses were performed to determine the independent risk factors for prostate cancer. The nomogram prediction model was generated using the finalized independent risk factors. Decision curve analysis (DCA) and the area under receiver operating characteristics curve (ROC) of both development group and validation group were calculated and compared to validate the accuracy and efficiency of the nomogram prediction model. Clinical utility curve (CUC) helped to decide the desired cut-off value for the prediction model. Comparison of the established nomogram with Prostate Cancer Prevention Trial Derived Cancer Risk Calculator (PCPT-CRC) and other domestic prediction models using the entire study population is conducted.

Results The independent risk factors determined through univariate and multivariate logistic regression analyses were: age, tPSA, fPSA, PV, DRE, TRUS and BMI. Nomogram prediction model was developed with the cut-off value of 0.31 . The AUC of development group and validation group were 0.856 and 0.797 respectively. DCA exhibits consistent observations with the findings. Through validating our prediction model as well as other three domestic prediction models based on the entire study population of 697 cases, our prediction model demonstrated significantly higher predictive value than all the other models.

Conclusion The nomogram for predicting prostate cancer can facilitate more accurate evaluation of the probability of having prostate cancer, and provide better ground for prostate biopsy.

Keywords Prostatic Neoplasms; Prostate-Specific Antigen; Nomograms

\section{Introduction}

With the prolongation of life span, the change of dietary structure and other factors, the incidence rate of prostate cancer is increasing over the years [1], making it more important to emphasize early screening for prostate cancer. In clinical practice, prostate biopsy is currently 
regarded as the gold standard for the diagnosis of prostate cancer. Whether to perform prostate puncture commonly depends on the level of serum prostate specific antigen (PSA) and its related parameters, digital rectal examination (DRE) and imaging results. However, the current primary non-invasive prostate cancer screening methods have resulted in an unsatisfactory amount of unnecessary prostate biopsy cases. Given the nature of being invasive and relatively expensive, prostate biopsy needs better ground to promote quality of life for patients. Many of the domestic pioneering scholars have proposed prostate cancer prediction models based on multiple clinical parameters in recent years, such as Tang et al. [2] (model 1), Huang et al. [3] (model 2), Li et al. [4] (model 3). There is a significant difference as for the morbidity rate of prostate cancer when we talk about different races or geographical distribution [1]. Therefore, prediction models which have been established from other regions such as PCPT-CRC [5] might not be fit for our study population. Meanwhile, the types of variables utilized for prediction model still need to be further explored, and the existing prediction models require more regional data analysis and validation. Based on the single center data source of Handan Central Hospital, this study aims to build a nomogram prediction model for prostate cancer, and promote early detection for patients with possible prostate cancer.

\section{Material and Methods}

Study population

In this study, data were retrospectively collected from a total amount of 789 patients who underwent transrectal ultrasound (TRUS)-guided transrectal prostate puncture $(12+\mathrm{X}$ core) in Handan Central Hospital from January 2014 to January 2020. All the prostate biopsies were performed by the experienced urologist from our department who has practiced his profession for more than 10 years. Pathological specimens were initially processed by 2 pathologists from the pathology department. Final pathological reports were provided by the senior pathologist after reviewing and the initial reports. Data screening was performed to exclude the following cases: First, 18 patients were previously diagnosed with prostate cancer or being surgically treated. Second, 35 patients were taking 5-alpha-reductase inhibitor/drug for treating endocrine dyscrasia in prostate cancer. Third, 15 patients had results of tPSA>100ng/mL. Forth, 24 cases had insufficient data. Eventually 697 cases were included. The median age is 71 years (40-95 years), and the median tPSA is $13.6 \mathrm{ng} / \mathrm{mL}(0.2-100 \mathrm{ng} / \mathrm{mL})$. To develop the nomogram, 548 
$80(80 \%)$ cases were randomly selected and rest of $149(20 \%)$ cases are set as the validation group 81 by SPSS software.

82 Ethics statement

83 The study was approved by ethics committee of Handan Central Hospital. Consent was obtained 84 from all patients prior to transrectal ultrasound-guided prostate puncture.

85 Methods

86 Baseline data were retrospectively collected from the clinical digital information system 87 including age, tPSA, fPSA, DRE results, TRUS findings, history of hypertension and diabetes. Prostate puncture was performed with one or more of the following criteria [6]: 1) tPSA $>10 \mathrm{ng} / \mathrm{ml}$; 2) $\% \mathrm{fPSA}<0.16$ when tPSA is within the range of $4 \sim 10 \mathrm{ng} / \mathrm{ml}$; 3) DRE and/or TRUS showed abnormal findings. PV was calculated by the formula: PV $(\mathrm{ml})=$ anterior/posterior diameter $(\mathrm{cm}) \times$ left/right diameter $(\mathrm{cm}) \times$ upper/lower diameter $(\mathrm{cm}) \times 0.52$. PSAD value was further calculated by dividing tPSA by PV. As an extended PSA-related figure, \%fPSA was calculated as well (fPSA/tPSA). BMI figures of all cases were calculated through weight (kg) divided by the square of height $(\mathrm{m} 2)$, and further sorted into four intervals according to the World Health Organization (WHO) guidelines for Asian population [7]: 56 underweight $(<18.5$ $\mathrm{kg} / \mathrm{m} 2)$ cases, 255 normal weight (18.5-22.9 kg/m2) cases, 266 overweight (23.0-27.4 kg/m2) cases, and 120 obese $(\geq 27.5 \mathrm{~kg} / \mathrm{m} 2)$ cases. Since there are only 56 underweight cases, in this study they were combined with normal weight group.

The three domestic prediction model equations are listed as follows:

Model 1[2]:

$\mathrm{e}^{-1.163+0.033 \mathrm{Age}+1.032 \mathrm{DRE}-2.821 \log \mathrm{PV}+2.292 \log \mathrm{PSA}}$

101

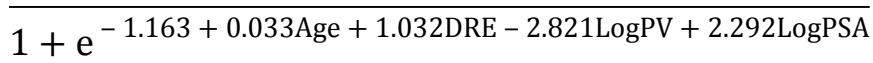

Model2[3]:

103

$\mathrm{e}^{-3.577+0.054(\text { Age })}-3.714(\mathrm{f} / \mathrm{tPSA})-1.324(\operatorname{Ln}(\mathrm{PV}))+0.977(\operatorname{Ln}(\mathrm{PSA}))+1.698($ DRE findings $)+0.458$ (hypoechoic) $1+\mathrm{e}^{-3.577+0.054(\mathrm{Age})-3.714(\mathrm{f} / \mathrm{tPSA})-1.324(\operatorname{Ln}(\mathrm{PV}))+0.977(\operatorname{Ln}(\mathrm{PSA}))+1.698(\mathrm{DRE} \text { findings })+0.458(\text { hypoechoic })}$

Model 3[4]:

105

$\mathrm{e}^{-5.348+0.09 \text { (Age) }-0.043(\mathrm{tPSA})+0.439(\mathrm{fPSA})-0.015(\mathrm{PV})-8.718(\mathrm{f} / \mathrm{tPSA})+2.614(\mathrm{PSAD})}$

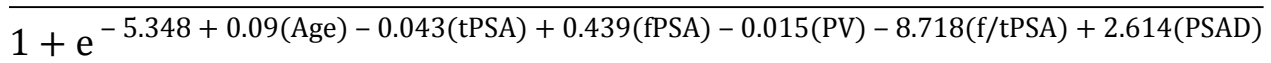

106

Statistical analysis

The Kolmogorov-Smirov test was used to assess the normal distribution of variables. For comparison purpose, T-test and U Mann-Whitney test were used to evaluate parametric and nonparametric continuous variables, respectively. Chi-squared test was used to compare 
110 qualitative variables. Univariate logistic regression analysis was utilized to determine the

111 independent risk factors. Multicollinearity diagnostics were conducted to determine whether the

112 independent factors have no multicollinearity (Variance Inflation Factor $<10$ ). The remaining

113 independent risk factors were included in the multivariate logistic regression analysis, and the

114 forward stepwise method was used to further determine the variables for developing the model.

115 The prediction model was graphically presented as a nomogram for clinical use. Clinical utility

116 curve was used to demonstrate the number of PCa cases which were left undiagnosed and the

117 number of non-PCa cases which were spared from unnecessary prostate biopsies. Then Youden

118 index was used to determine the desired cut-off value. Diagnostic accuracy was quantified as the

119 area under the ROC curve (AUC). Statistical differences between AUCs were compared using

120 the DeLong method. Based on the optimal cut-off value, the diagnostic accuracy was assessed

121 using sensitivity, specificity, positive predictive value (PPV) and negative predictive value

122 (NPV). The calibration curve was utilized to assess the agreement of the nomogram-predicted

123 probability with the actual observed probability. Decision curve analysis (DCA) was conducted

124 to determine the net benefit derived from the use of the created nomogram. SPSS 22.0,

125 medcalc19.0.7 and R language 4.0.0 statistical software were used to process the data. A $P$-value

$126<0.05$ is considered statistically significant.

127

128

Results

129 Patient demographics

130 Out of a total amount of 697 cases, 504 (72.3\%) cases were non-prostate cancer patients, 193

$131(27.7 \%)$ cases were found with prostate cancer according to the biopsy results. Specifically in

132 non-prostate cancer group, there are 454 cases of benign prostatic hyperplasia, 36 cases of

133 prostatic intraepithelial neoplasia, and 14 cases of prostatitis. Comparison of the baseline data

134 indicated that there is no significant difference between the development and validation group in

135 the general situation of patients, characters of age, tPSA, fPSA, and other indicators $(\mathrm{P}>0.05)$

136 (Table 1). In normal weight, overweight and obese BMI intervals, the rate of having prostate

137 cancer is $22.5 \%(70 / 311), 29.3 \%(78 / 266)$, and $37.5 \%(45 / 120)$ respectively, indicating a

138 statistical difference among the three groups $\left(\chi^{2}=10.293, \mathrm{P}<0.05\right)$

139 Nomogram development

140 Univariate logistic regression analysis of the development group showed that age, tPSA, fPSA, 
141 PV, DRE, TRUS, BMI were statistically significant $(P<0.05)$, whereas hypertension and diabetes

142 were not significantly related to prostate cancer. Multicollinearity diagnostics were performed 143 utilizing the abovementioned independent risk factors, and the VIFs were 1.042, 2.338, 2.405, $1441.121,1.061,1.043,1.022$ respectively, suggesting that there was no multicollinearity among the 145 seven independent risk factors. Lastly, multivariate logistic regression confirmed that age, tPSA, 146 fPSA, PV, DRE, TRUS, and BMI served as independent risk factors for prostate cancer $(P<0.05)$ 147 which could be included in the development of predicting model (Table 2).

148 Based on the multivariate logistic regression analysis, the predicting equation was generated with 149 the calculated coefficients: $\operatorname{logit}(1 / 1-\mathrm{P})=-4.934+0.038 \times$ Age $+0.025 \times \mathrm{tPSA}+0.195 \times \mathrm{fPSA}-$ $0.028 \times \mathrm{PV}+1.158 \times \mathrm{DRE}+1.517 \times \mathrm{TRUS}+0.617 \times \mathrm{BMI}$ (Table 3$)$. According to the variables and their corresponding regression coefficients, the nomogram representing the predicting model is then established (Figure 1).

To properly use the nomogram, points are collected from each category (age, tPSA, fPSA, PV, DRE, TRUS and BMI) according to an individual's corresponding test results, and the total points indicate a diagnostic possibility of having prostate cancer. Nomogram validation The clinically commonly used parameters tPSA, f/tPSA, and PSAD are used to determine the diagnostic value of the prediction model through comparing their ROC curves. The AUC values of development group, tPSA, \%fPSA, PSAD were $0.856,0.713,0.624,0.761$ respectively (Figure 2, Table 3), and the AUC values of validation group, tPSA, \%fPSA and PSAD were 0.797, 0.662, 0.624, 0.673 respectively (Figure 3, Table 4). Predicting model indicated statistically significant better diagnostic value against other risk factors in both development group and validation group $(P<0.05)$. Clinical utility curve (CUC) demonstrated the dynamics between the percentage of cases left undiagnosed and percentage of cases saved from unnecessary biopsies at any threshold of probabilities (Figure 8, Figure 9). The cut-off value for the prediction model was selected as 0.31 when Youden index reached maximum value. Sensitivity, specificity, positive predictive value, negative predictive value, false negative rate and false positive rate were $73 \%, 85.8 \%, 65.5 \%, 89.6 \%, 27 \%$ and $14.2 \%$ respectively. Applying the cut-off value 0.31 into the validation group resulted the sensitivity, specificity, positive predictive value, negative predictive value, false negative rate and false positive rate, which were $62.2 \%, 87.5 \%, 68.3 \%, 84.3 \%, 37.8 \%$ and $12.5 \%$ respectively (Table 4 ). 
172 The calibration plot demonstrated an outstanding correlation between the predicted and actual

173 probability in both development group and validation group, in which the predicted probability

174

175

176

177

178

179

180

181

182

183

184

185

186

187

188

189

190

191

192

193

194

195

196

197

198

199

200

201

202 and actual probability lines are closely aligned with remarkable $P$-values of 0.374 in development group and 0.236 in validation group. The intercept and slope in the development group were -0.057 and 1.044, while in the validation group were 0.178 and 0.877 (Figure 4, Figure 5).

When the threshold range is within $11 \% \sim 69 \%$ and $75 \% \sim 81 \%$ in the validation group, and $4 \% \sim 83 \%$ in the development group, the net benefit of our prediction model is higher than that of the other clinically commonly used parameters (Figure 6, Figure 7). The cut-off value of 0.31 which resulted from ROC curve analysis was utilized as the determined threshold probability. The net benefit and net reduction of our prediction model are both higher than that of the other three diagnostic parameters in both development and validation groups (Table 5).

Together with the other three domestic prediction models, our developed prediction model is further validated through utilizing the validation group of 149 cases. The AUC of our prediction model, domestic model 1, domestic model 2 and domestic model 3 are 0.797, 0.739, 0.753 and 0.694, respectively. Separately, enable to compare with PCPT-CRC, the validation group was modified according to the limitations for entries of PCPT-CRC. Specifically, 1 case was exempted for being over 55 years old, 12 cases were ruled out for having tPSA more than $50 \mathrm{ng} / \mathrm{mL}$, resulting a separate validation group with 136 cases. The AUC of our prediction model and PCPT-CRC using such new validation group were 0.793 and 0.668 (table 6). Statistically significant differences were found comparing our prediction model with PCPT-CRC and domestic prediction model $3(P<0.05)$. However, it was not significantly different compared with domestic model 1 and 2 (table 7).

\section{Discussion}

Prostate biopsy is the gold standard for diagnosing prostate cancer, but there are risks of complications such as hematuria, bloody stool, urinary retention and infection. Therefore, various international researchers intended to reduce prostate puncture rate through utilizing different types of variables to develop predicting models and presenting them as forms of classification tree model [8], artificial neural network model [9] and nomogram model for better comprehension and feasibility. Among the presenting models, nomogram model has the

Peerj reviewing PDF | (2021:11:67853:1:0:NEW 8 Jan 2022) 
203 characteristics of integrating multiple predictive variables, quantifying the contribution of related

204 risk factors, and presenting the predicting model in geometric form for better accessibility and

205 utility. Even though many facilities have established their predicting models such as Prostate

206 Cancer Prevention Trial Derived Cancer Risk Calculator (PCPT-CRC) [5] and the Montreal

207 (Canada) prediction model [10], it is not directly suitable for prostate cancer screening in

208 Chinese population due to demographic differences [11]. Meanwhile, people of same race living

209 in different regions could lead to a difference in the incidence rate of prostate cancer [12]. Our

210 statistical analysis of comparing with PCPT-CRC further confirmed this observation. Therefore,

211 it is of great significance to establish a prediction model for prostate cancer based on the

212 population from a specific region.

213 Several domestic prediction models based on PSA and its related clinical parameters were

214 established in China. Tang et al. [2] established a prediction model integrating age, PSA, PV, and

215 DRE with a total population of 535 cases, and the AUC was 0.848. Another domestic prediction

216 model which developed by Huang et al. [3] included age, PSA, PV, \%fPSA, TRUS and DRE as

217 independent risk factors, acquired the AUC of 0.853 based on a total population of 1104 cases.

218 Lastly, with the population of 958 cases, Li et al. [4] established a prediction model based on age,

219 tPSA, fPSA, PV, \%fPSA and PSAD, resulting the AUC of 0.854. In this study, univariate and

220 multivariate logistic regression analysis showed that age, TPSA, fPSA, PV, DRE, TRUS and

221 BMI were independent predictors of prostate cancer in the development group. Based on these

222 clinical variables, a nomogram prediction model was established. Considering the progression of

223 prostate cancer when false negative results happen, versus the complications which prostate

224 puncture possibly brings, we have decided to take the cut-off value of AUC when the Youden

225 index was at its optimal level. Such cut-off value of AUC in the development group was 0.31.

226 The sensitivity, specificity, positive predictive value, negative predictive value, false negative

227 rate, false positive rate and AUC value of development group were 73\%, 85.8\%, 65.5\%, 89.6\%,

$22827 \%, 14.2 \%$ and 0.856 , respectively. By applying the cut-off value 0.31 into the validation group

229 resulted sensitivity, specificity, positive predictive value, negative predictive value, false

230 negative rate, false positive rate and AUC value were calculated as $62.2 \%, 87.5 \%, 68.3 \%, 84.3 \%$,

$23137.8 \%, 12.5 \%$ and 0.797 , respectively, which were significantly higher than PSA related

232 parameters. By integrating the threshold of 0.31 in the development group, our prediction model

233 saved $85.8 \%$ of unnecessary prostate biopsies with having $27 \%$ of missed positive cases. 
234 Therefore, prostate biopsy is recommended when the prediction probability is greater than 0.31 , 235 otherwise active monitoring is preferred. After the analyses of calibration curve and decision

236

237

238

239

240

241

242

243

244

245

246

247

248

249

250

251

252

253

254

255

256

257

258

259

260

261

262

263

264 curve, the development group and validation group showed that the prediction model had high predictive ability and clinical practicability, from which decision curve analysis indicated that utilizing our prediction model to determine the necessity of conducting prostate biopsy could significantly increase the net benefit while decreasing the rate of unnecessary prostate biopsies compared with other currently commonly used clinical diagnostic parameters. Compared with other three domestic prediction models, our prediction model demonstrated a significantly higher predictive value through validating over the entire study population. This finding suggests that: 1) even with the same race, living in different regions might lead to some distinctively different profiles of prostate cancer, making it difficult for a prediction model which was developed in a specific region to maintain its predictive value in other regions, even towards people with the same race; 2) Inclusion criteria were not always identical among different prediction models; 3) different types and amount of independent risk factors included in the development of nomogram prediction model might eventually alter the predictive value. The clinical variables included in the nomogram of this study are currently commonly used in prostate screening routines. Integrating and analyzing them in a wholistic manner can further improve the accuracy of prostate cancer detection and reduce unnecessary prostate puncture without additional medical costs.

The relationship between obesity and prostate cancer is not clear. Some researchers believe that obesity is a risk factor for prostate cancer [13], and it can increase the mortality rate of prostate cancer patients [14]. Among different BMI intervals, statistical analysis showed that the detection rate of prostate cancer increased with the increasement of BMI level, suggesting that obesity is likely to increase the risk of prostate cancer. The possible reasons for this phenomenon are: (1) obesity can lead to chronic inflammation, which may be related to a variety of cancers, including prostate cancer [15] (2) Obesity can lead to high levels of insulin and insulin-like receptor factor-1, which are closely related to the development of tumor [16]. (3) Leptin is mainly secreted by adipose tissue [17], and the increasement of leptin level is related to tumor invasion [18] (4) BMI is negatively correlated with PSA [19], resulting in delayed detection of prostate cancer in obese people. After regression analysis, BMI was included as an independent predictor in the nomogram, which further confirmed that there was a correlation between obesity 
265

266

267

268

269

270

271

272

273

274

275

276

277

278

279

280

281

282

283

284

285

286

287

288

289

290

291

292

293

294

295

296

297

298

299

300

and prostate cancer.

In order to improve the accuracy of prostate cancer prediction model, researchers have tried to incorporate more clinical variables into their prediction models. Zhu et al [20]. established a nomogram model for predicting prostate cancer based on Prostate Health Index (PHI), and some other researchers integrated prostate imaging reporting and data system version 2 (PI-RADSv2) into their prediction model [21]. These innovative models showed great predictive values.

However, due to the uneven development level of medical care in China, the detection of p2PSA has not been widely put into practice. Besides, image grading is a subjective evaluation method, and there may be different judgements from different experts. By comparing the prediction accuracy of four models from different origins, Wang Li et al [22]. found that the prediction accuracy of Huang et al [3]. had no difference with that of PCPT-CRC model [5] and Montreal model [10], but whether there was any consistency between different races and different regions was worth further discussion. Compared with the abovementioned models, the clinical variables included in this nomogram prediction model do not need complex calculation or additional equipment, making it possible to benefit both outpatients and inpatients. At the same time, we encourage more imaging results as independent variables to be included in the nomogram prediction model and multi-center large sample cross validation of different races and regions, so as to further improve the accuracy of the nomogram prediction model.

Shortcomings: (1) this is a retrospective study, hence there may lead to unavoidable selection bias; (2) the prediction model lacks external validation; (3) the sample size of single-center study is relatively small, and further multi-center joint study is needed to validate on a larger scale.

\section{Reference}

[1] Siegel RL and Miller KD. Cancer Statistics, 2021. 2021; 71: 7-33.

[2] Tang P, Chen H, Uhlman M, Lin YR, Deng XR, Wang B, Yang WJ and Xie KJ. A nomogram based on age, prostate-specific antigen level, prostate volume and digital rectal examination for predicting risk of prostate cancer. Asian J Androl 2013; 15: 129-133.

[3] Huang Y, Cheng G, Liu B, Shao P, Qin C, Li J, Hua L and Yin C. A prostate biopsy strategy based on a new clinical nomogram reduces the number of biopsy cores required in highrisk patients. BMC Urol 2014; 14: 8.

[4] Li FL, Li WD, Zhang YY, Cui L, Yin XT, Yin ZHY, Qi SY, Xu Y, Guo G, Tang J, Shi

HY, Gao JP and Zhang X. Development of a nomogram for predicting the risk of positive prostate biopsy. Chin J Urol 2016; 37: 616-620.

[5] Thompson IM, Ankerst DP, Chi C, Goodman PJ, Tangen CM, Lucia MS, Feng Z, Parnes $\mathrm{HL}$ and Coltman CA, Jr. Assessing prostate cancer risk: results from the Prostate Cancer Prevention Trial. J Natl Cancer Inst 2006; 98: 529-534.

[6] Sun YH. Chinese expert consensus on prostate biopsy. Chin J Urol 2016; 37: 241-244. 
301

302

303

304

305

306

307

308

309

310

311

312

313

314

315

316

317

318

319

320

321

322

323

324

325

326

327

328

329

330

331

332

333

334

335

336

337

338

339

340

341

342

343

344

345

346

[7] Feng R, Chang ET, Liu Z, Liu Q, Cai Y, Zhang Z, Chen G, Huang QH, Xie SH, Cao SM, Zhang Y, Yun J, Jia WH, Zheng Y, Liao J, Chen Y, Lin L, Ernberg I, Huang G, Zeng Y, Zeng YX, Adami HO and Ye W. Body mass index, body shape, and risk of nasopharyngeal carcinoma: A population-based case-control study in Southern China. Cancer Med 2019; 8: 1835-1844.

[8] Eifler JB, Feng Z, Lin BM, Partin MT, Humphreys EB, Han M, Epstein JI, Walsh PC, Trock BJ and Partin AW. An updated prostate cancer staging nomogram (Partin tables) based on cases from 2006 to 2011. BJU Int 2013; 111: 22-29.

[9] Cai B and Jiang X. A novel artificial neural network method for biomedical prediction based on matrix pseudo-inversion. J Biomed Inform 2014; 48: 114-121.

[10] Karakiewicz PI, Benayoun S, Kattan MW, Perrotte P, Valiquette L, Scardino PT, Cagiannos I, Heinzer H, Tanguay S, Aprikian AG, Huland H and Graefen M. Development and validation of a nomogram predicting the outcome of prostate biopsy based on patient age, digital rectal examination and serum prostate specific antigen. J Urol 2005; 173: 1930-1934.

[11] Wu YS, Zhang N, Liu SH, Xu JF, Tong SJ, Cai YH, Zhang LM, Bai PD, Hu MB, Jiang HW, Na R, Ding Q and Sun YH. The Huashan risk calculators performed better in prediction of prostate cancer in Chinese population: a training study followed by a validation study. Asian $\mathrm{J}$ Androl 2016; 18: 925-929.

[12] Gu CY, Qin XJ, Huang YQ, Zhu Y, Dai B and Ye DW. [The prostate cancer precision screening program: a preliminary report after recruitment of 2159 men]. Zhonghua Yi Xue Za Zhi 2019; 99: 3292-3297.

[13] Cao Y and Giovannucci E. Obesity and Prostate Cancer. Recent Results Cancer Res 2016; 208: $137-153$.

[14] Dickerman BA, Ahearn TU, Giovannucci E, Stampfer MJ, Nguyen PL, Mucci LA and Wilson KM. Weight change, obesity and risk of prostate cancer progression among men with clinically localized prostate cancer. Int J Cancer 2017; 141: 933-944.

[15] Thapa D and Ghosh R. Chronic inflammatory mediators enhance prostate cancer development and progression. Biochem Pharmacol 2015; 94: 53-62.

[16] Xue M, Cao X, Zhong Y, Kuang D, Liu X, Zhao Z and Li H. Insulin-like growth factor-1 receptor (IGF-1R) kinase inhibitors in cancer therapy: advances and perspectives. Curr Pharm Des 2012; 18: 2901-2913.

[17] Garcia-Galiano D, Borges BC and Allen SJ. PI3K signalling in leptin receptor cells: Role in growth and reproduction. 2019; 31: e12685.

[18] Candelaria PV, Rampoldi A, Harbuzariu A and Gonzalez-Perez RR. Leptin signaling and cancer chemoresistance: Perspectives. World J Clin Oncol 2017; 8: 106-119.

[19] Aref AT, Vincent AD, O'Callaghan ME, Martin SA, Sutherland PD, Hoy AJ, Butler LM and Wittert GA. The inverse relationship between prostate specific antigen (PSA) and obesity. Endocr Relat Cancer 2018; 25: 933-941.

[20] Zhu Y, Han CT, Zhang GM, Liu F, Ding Q, Xu JF, Vidal AC, Freedland SJ, Ng CF and Ye DW. Development and external validation of a prostate health index-based nomogram for predicting prostate cancer. Sci Rep 2015; 5: 15341.

[21] Niu XK, He WF, Zhang Y, Das SK, Li J, Xiong Y and Wang YH. Developing a new PIRADS v2-based nomogram for forecasting high-grade prostate cancer. Clin Radiol 2017; 72: 458-464.

[22] Wang L, Li G, Xie GS, Zhang XF, Yin HM, Hu Q, Chen H, Pu JX and Hou JQ. An external validation and comparison of the predictive accuracy of four models designed to predict the probability of a positive prostate biopsy. Chin J Urol 2016; 37: 507-510. 
Figure 1

Nomogram for prostate cancer prediction model

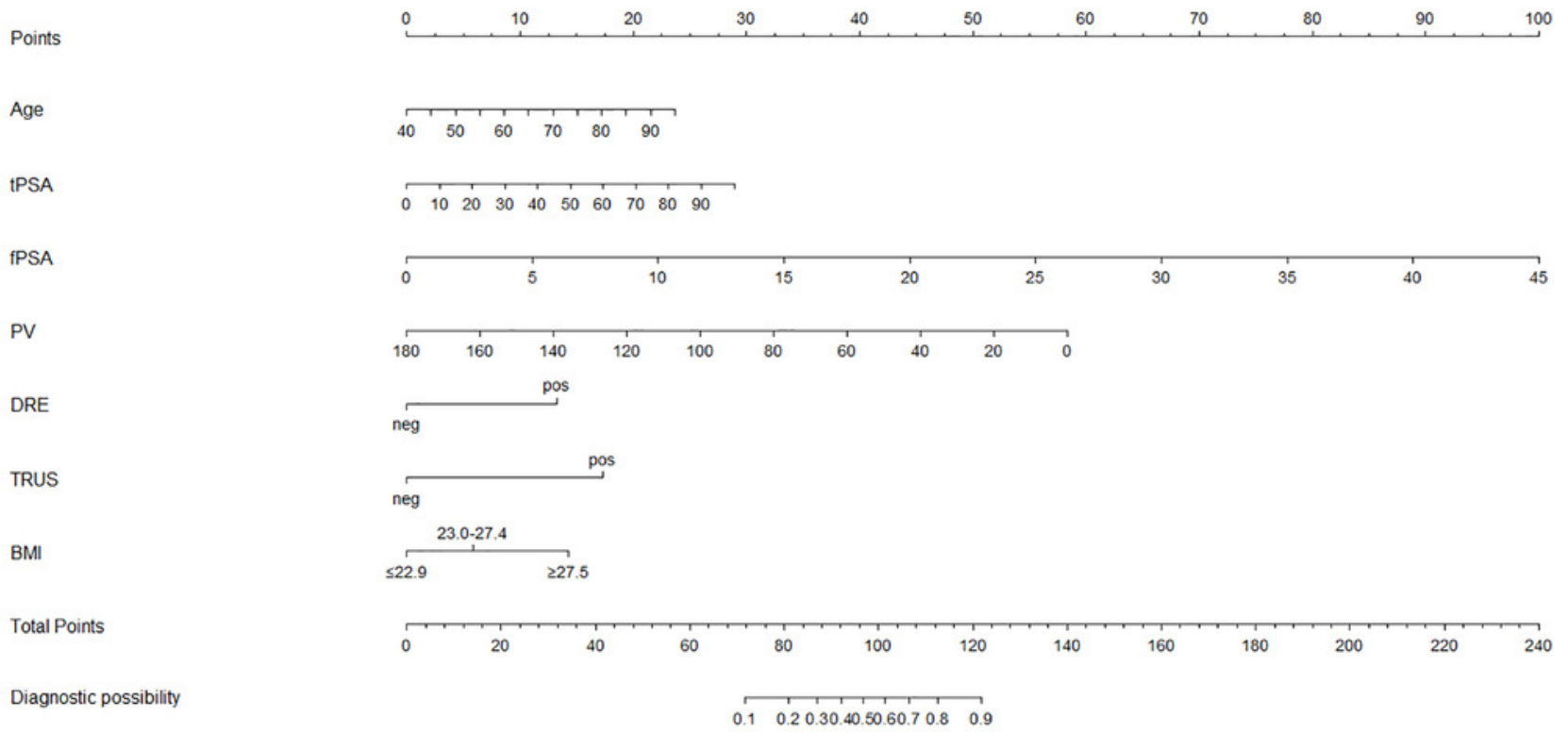


Figure 2

ROC curve presenting the discrimination power of the nomogram (development group)

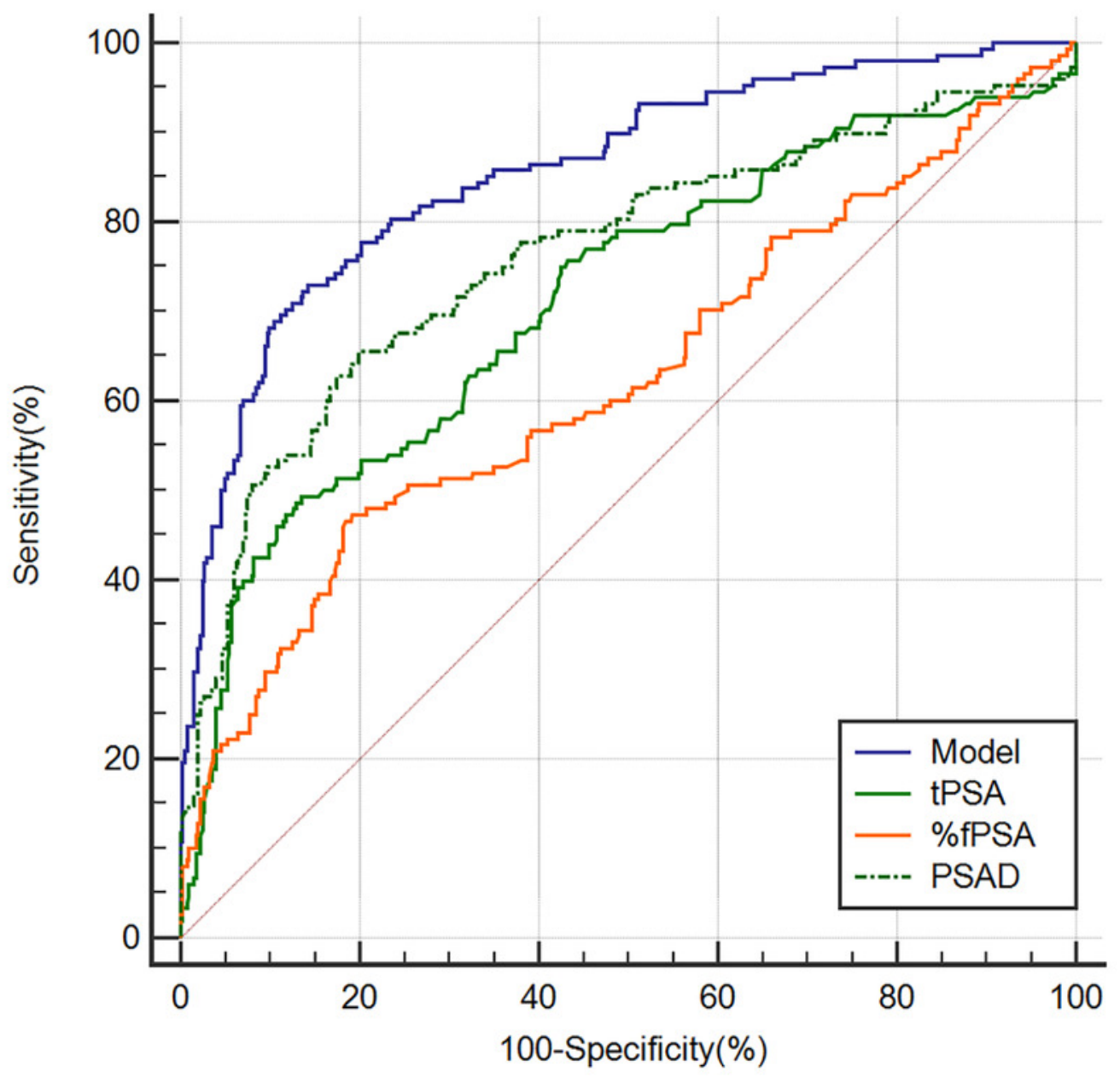


Figure 3

ROC curve presenting the discrimination power of the nomogram (validation group)

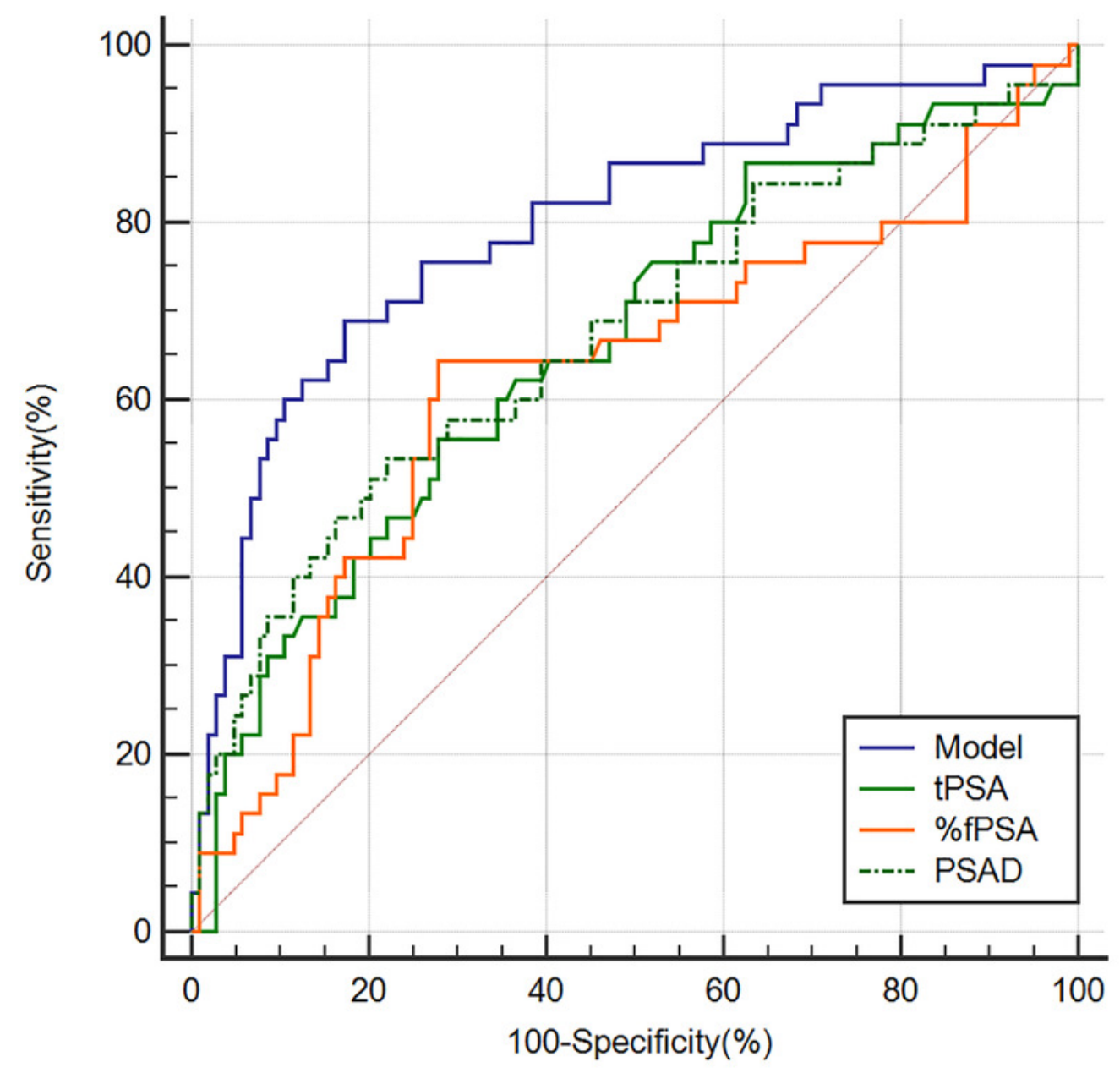


Figure 4

Calibration curve of the prediction model in the development group

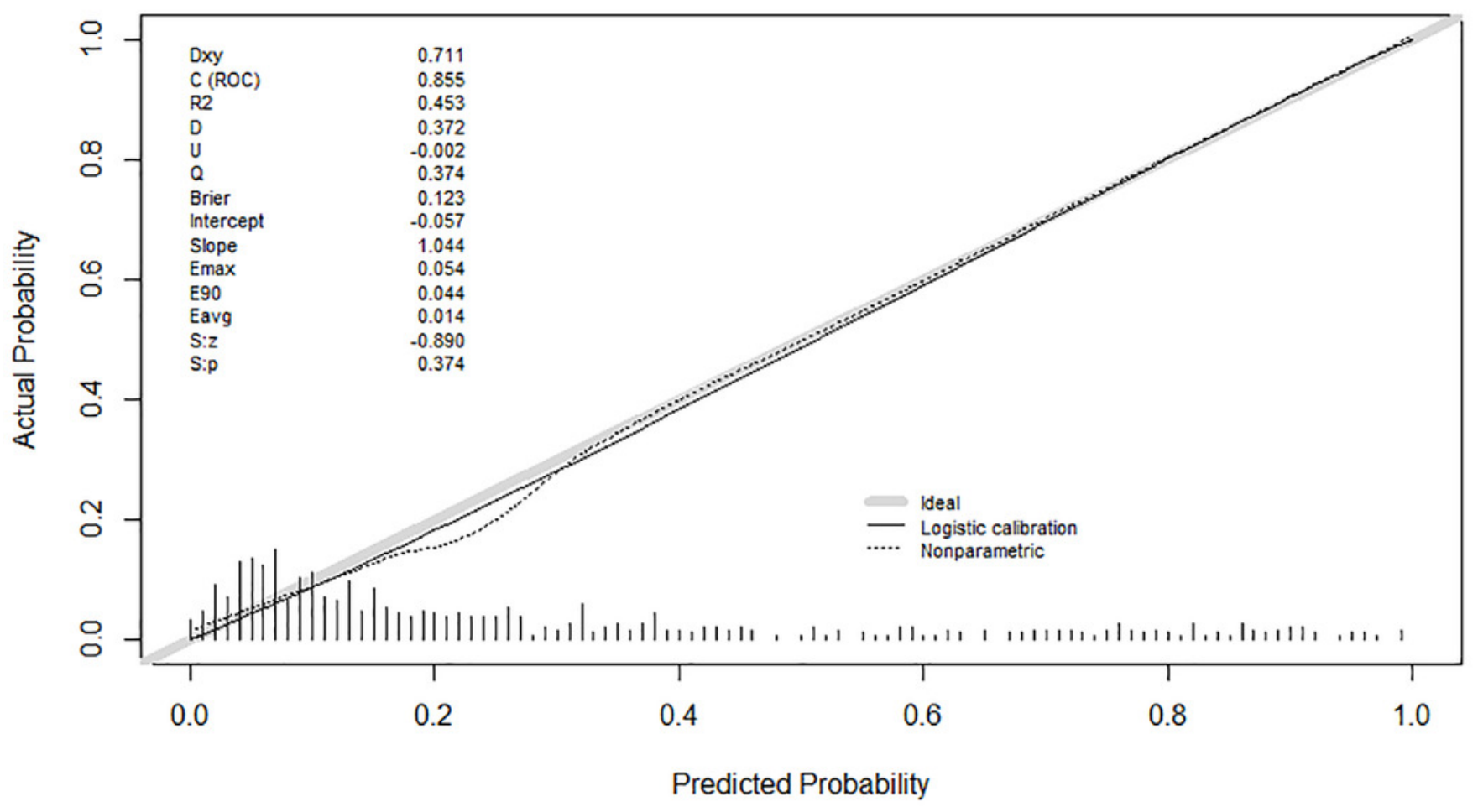


Figure 5

Calibration curve of the prediction model in the validation group

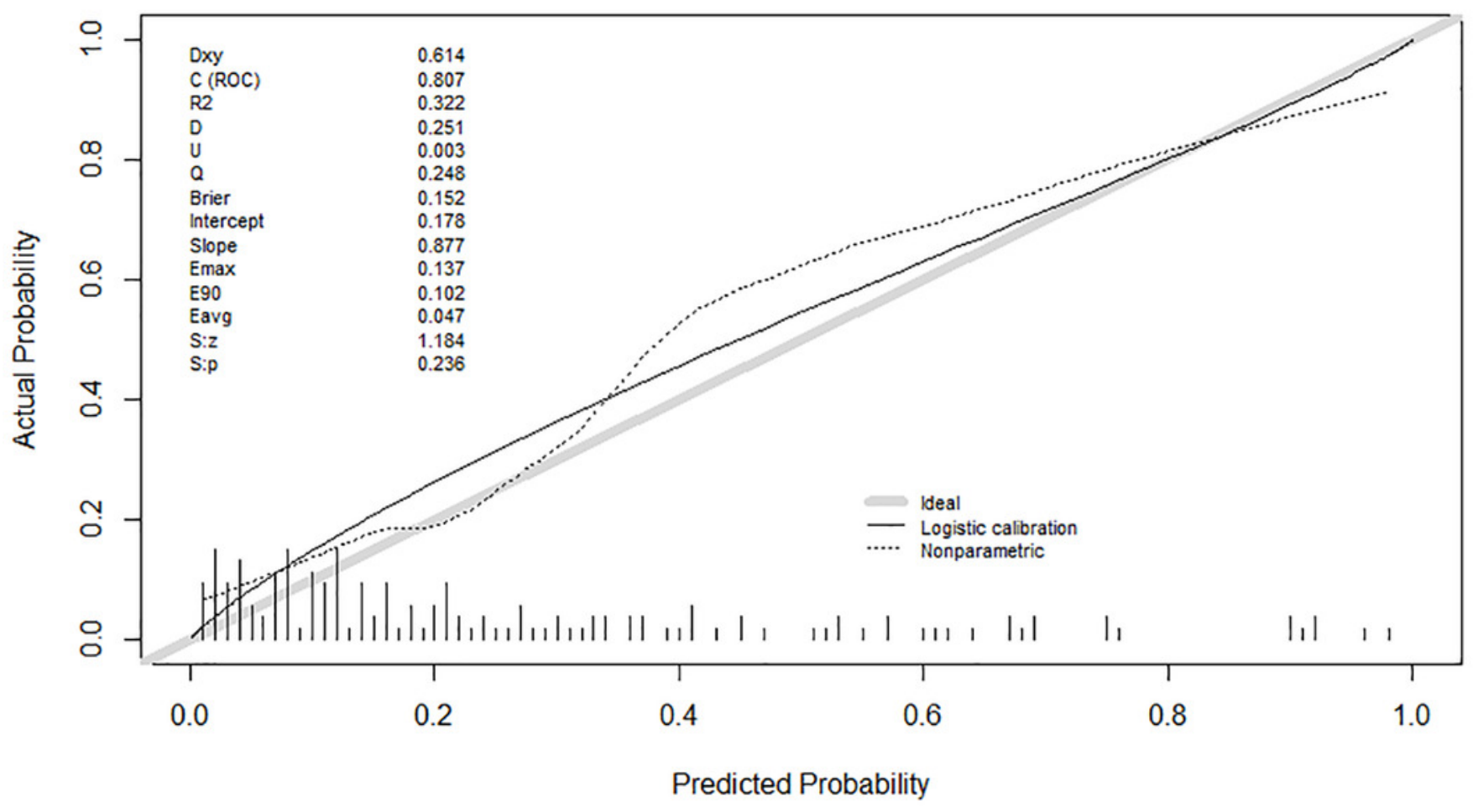


Figure 6

Decision curve analysis of the prediction model and other variables in the development group

predmodelA: prediction model; predmodelB: tPSA; predmodelC: \%fPSA; predmodelD: PSAD

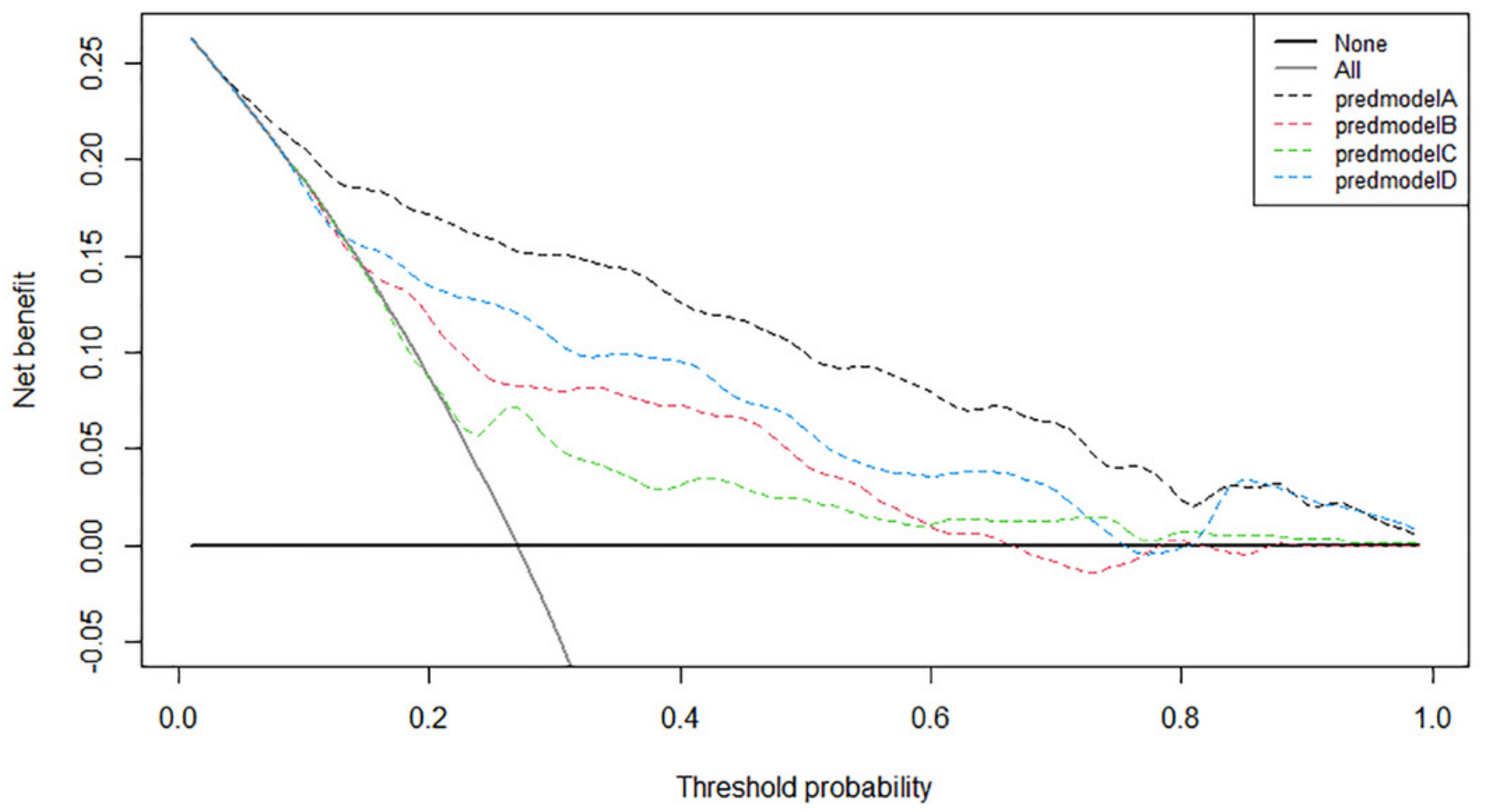


Figure 7

Decision curve analysis of the prediction model and other variables in the validation group

predmodelA: prediction model; predmodelB: tPSA; predmodelC: \%fPSA; predmodelD: PSAD

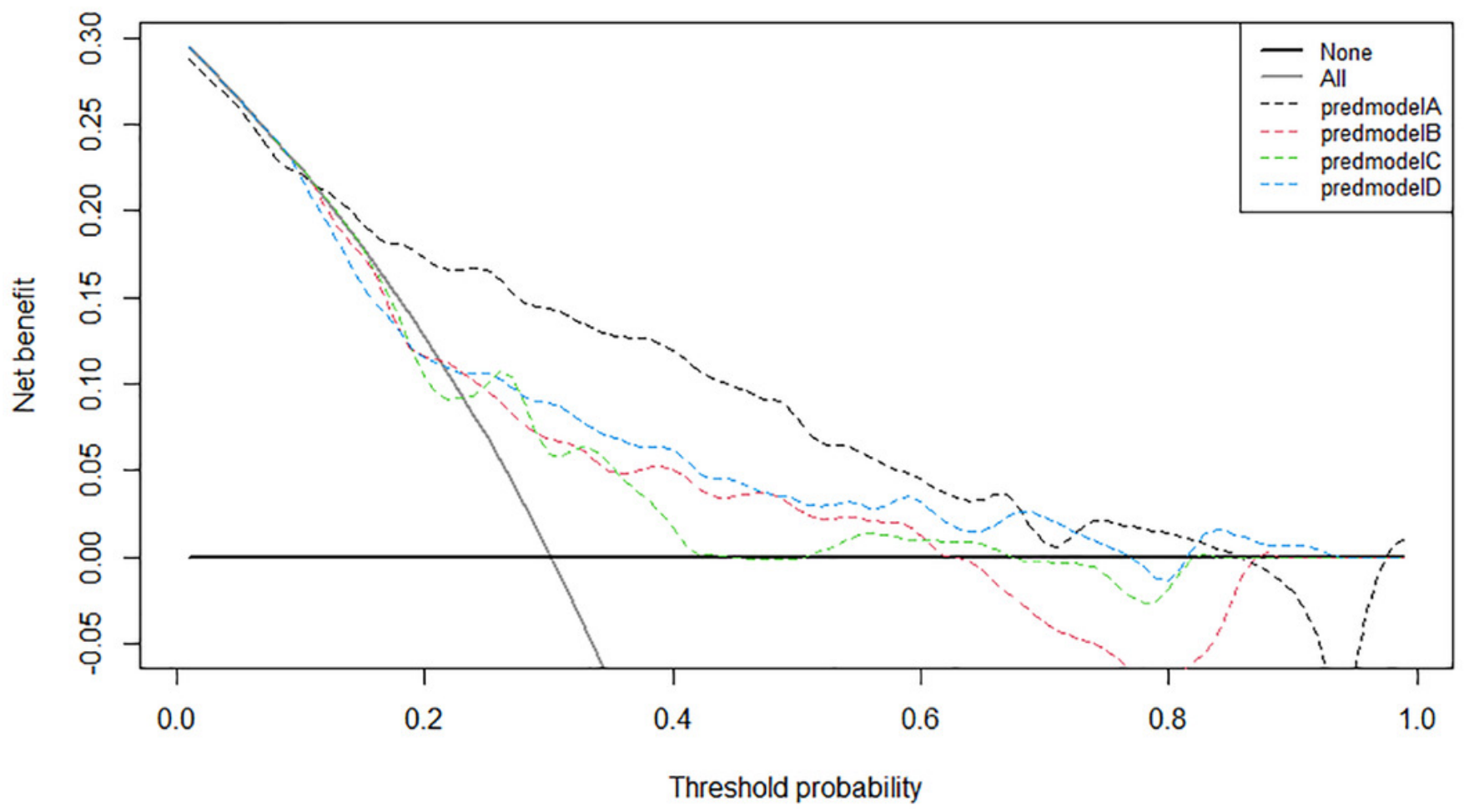


Figure 8

Clinical utility curve of the development group

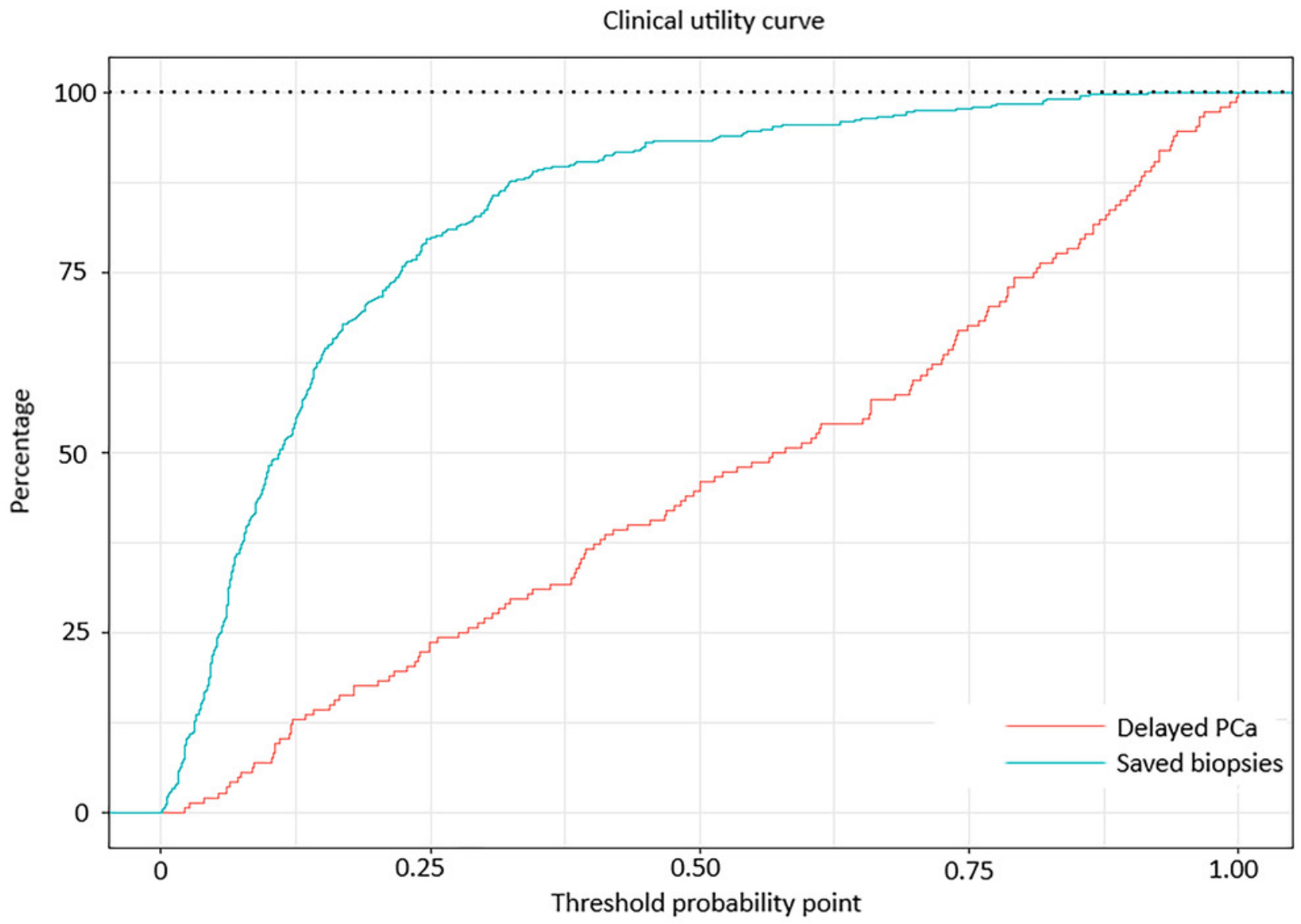


Figure 9

Clinical utility curve of the validation group

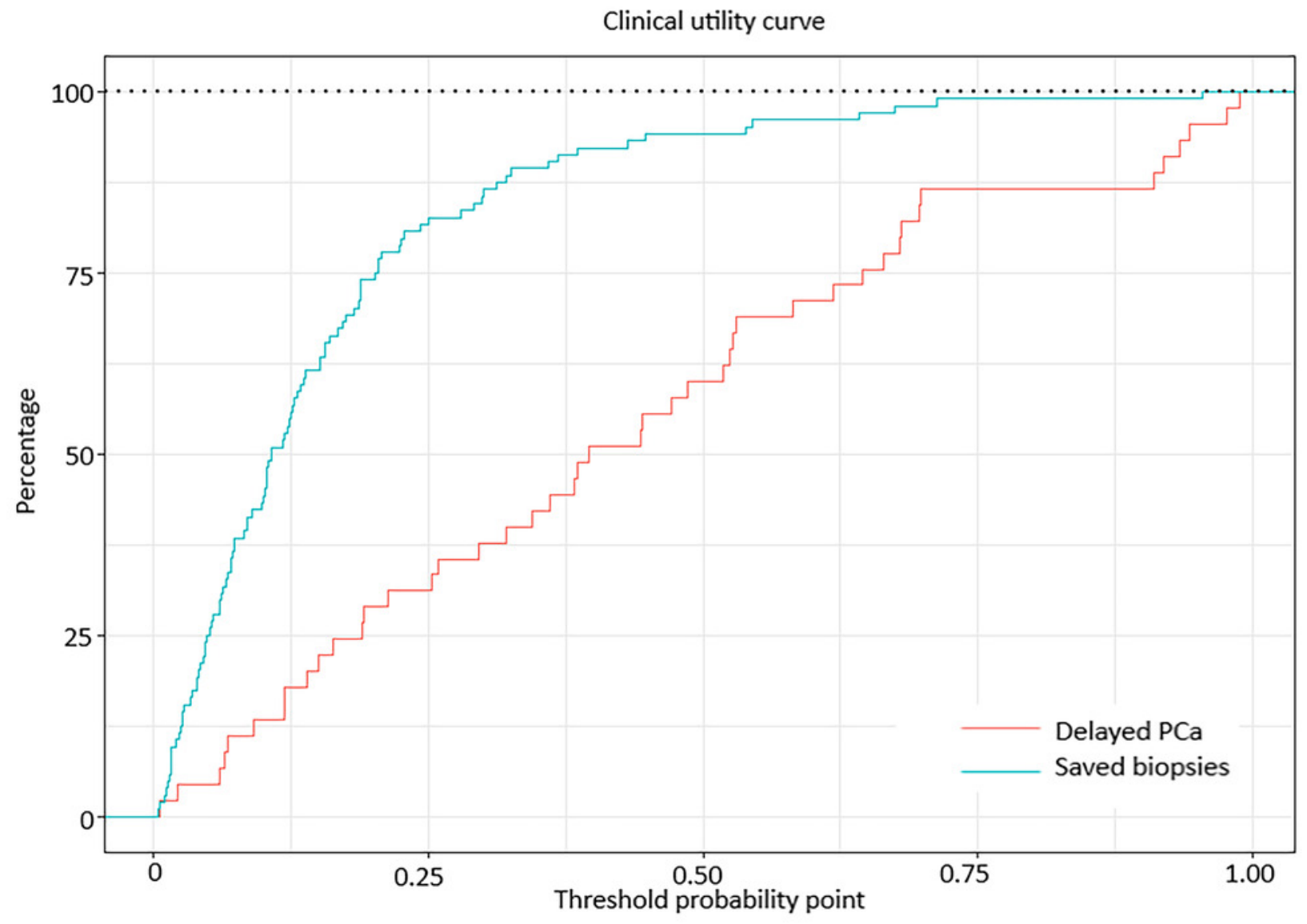




\section{Table $\mathbf{1}$ (on next page)}

Baseline clinical characteristics of the development group and validation group

Abbreviations: tPSA, total prostate-specific antigen; fPSA, free prostate-specific antigen; PV, prostate volume; DRE, digital rectal examination; TRUS, transrectal ultrasound; *Lowechogenicity in the peripheral zone of the prostate was defined as 'positive'; other findings were defined as 'negative'. 
1

\begin{tabular}{|c|c|c|c|c|c|}
\hline Parameter & Total & Development group & Validation group & $\mathrm{Z} / \chi^{2}$ & P-value \\
\hline Number of patients & 697 & 548 & 149 & & \\
\hline Age(year) & $71(66 \sim 77)$ & $71(66 \sim 77)$ & $72(65 \sim 78)$ & -0.751 & 0.453 \\
\hline $\operatorname{tPSA}(\mathrm{ng} / \mathrm{ml})$ & $13.6(5.6 \sim 30.7)$ & $13.6(5.6 \sim 30.5)$ & $14.6(5.4 \sim 32.8)$ & -0.279 & 0.78 \\
\hline $\mathrm{fPSA}(\mathrm{ng} / \mathrm{ml})$ & $1.8(0.8 \sim 4.3)$ & $1.8(0.73 \sim 4.2)$ & $2.0(0.95 \sim 4.5)$ & -1.071 & 0.284 \\
\hline $\mathrm{PV}(\mathrm{ml})$ & $47.9(36.0 \sim 69.2)$ & $46.6(35.6 \sim 68.2)$ & $50.4(38.1 \sim 72.4)$ & -1.556 & 0.12 \\
\hline$\%$ fPSA & $0.14(0.11 \sim 0.18)$ & $0.13(0.1 \sim 0.17)$ & $0.15(0.12 \sim 0.20)$ & -3.088 & 0.002 \\
\hline PSAD & $0.26(0.1 \sim 0.60)$ & $0.25(0.11 \sim 0.61)$ & $0.26(0.09 \sim 0.55)$ & -0.377 & 0.706 \\
\hline $\operatorname{DRE}[\mathrm{n}(\%)]$ & & & & 0.046 & 0.83 \\
\hline Normal & 557 & $437(80)$ & $120(81)$ & & \\
\hline Suspect cancer & 140 & $111(20)$ & 29(19) & & \\
\hline TRUS finging* $[\mathrm{n}(\%)]$ & & & & 0.47 & 0.493 \\
\hline Negative & $475(68)$ & $370(68)$ & $105(70)$ & & \\
\hline Positive & $222(32)$ & $178(32)$ & $44(30)$ & & \\
\hline $\operatorname{BMI}\left(\mathrm{kg} / \mathrm{m}^{2}\right)[\mathrm{n}(\%)]$ & & & & 1.959 & 0.376 \\
\hline$\leqslant 22.9$ & $311(45)$ & $237(43)$ & $74(50)$ & & \\
\hline $23.0 \sim 27.4$ & $266(38)$ & $214(39)$ & $52(35)$ & & \\
\hline$\geqslant 27.5$ & $120(17)$ & $97(18)$ & $23(15)$ & & \\
\hline Hyertension[n(\%)] & & & & 0.803 & 0.37 \\
\hline No & $322(46)$ & $258(47)$ & $64(43)$ & & \\
\hline Yes & $375(54)$ & $290(53)$ & $85(57)$ & & \\
\hline Diabetes $[n(\%)]$ & & & & 0.256 & 0.613 \\
\hline No & $575(82)$ & $450(82)$ & $125(84)$ & & \\
\hline Yes & $122(18)$ & $98(18)$ & $24(16)$ & & \\
\hline
\end{tabular}

2 


\section{Table 2 (on next page)}

Univariate and multivariate logistic regression models in the development group

Abbreviations: tPSA, total prostate-specific antigen; fPSA, free prostate-specific antigen; PV, prostate volume; DRE, digital rectal examination; TRUS, transrectal ultrasound; OR, odds radio; $\mathrm{Cl}$, confidence interval. 
1

\begin{tabular}{ccccc}
\hline \multirow{2}{*}{ Variable } & \multicolumn{2}{c}{ Univariate analysis } & \multicolumn{2}{c}{ Multivariate analysis } \\
\cline { 2 - 5 } & OR(95\%CI) & P-value & OR(95\%CI) & P-value \\
\hline Age & $1.036(1.010 \sim 1.063)$ & 0.007 & $1.039(1.005 \sim 1.074)$ & 0.024 \\
tPSA & $1.040(1.030 \sim 1.050)$ & 0.01 & $1.026(1.010 \sim 1.042)$ & 0.002 \\
fPSA & $1.256(1.187 \sim 1.233)$ & $<0.001$ & $1.215(1.106 \sim 1.336)$ & $<0.001$ \\
PV & $0.987(0.979 \sim 0.995)$ & 0.01 & $0.972(0.961 \sim 0.983)$ & $<0.001$ \\
DRE & $3.131(2.024 \sim 4.843)$ & $<0.001$ & $3.185(1.798 \sim 5.641)$ & $<0.001$ \\
TRUS & $4.1(2.754 \sim 6.104)$ & $<0.001$ & $4.560(2.773 \sim 7.500)$ & $<0.001$ \\
BMI & $1.466(1.137 \sim 1.889)$ & 0.003 & $1.852(1.337 \sim 2.567)$ & $<0.001$ \\
Hypertension & $0.988(0.677 \sim 1.442)$ & 0.951 & & \\
Diabetes & $0.853(0.515 \sim 1.412)$ & 0.536 & &
\end{tabular}

2 


\section{Table 3(on next page)}

Result of multivariate logistic regression analysis in the development group

Abbreviations: tPSA, total prostate-specific antigen; fPSA, free prostate-specific antigen; PV, prostate volume; DRE, digital rectal examination; TRUS, transrectal ultrasound; SE, Standard error; OR, odds radio; $\mathrm{Cl}$, confidence interval. 
1

\begin{tabular}{ccccccc}
\hline Variable & Coefficient & SE & Wald & OR & $95 \%$ CI & P-value \\
\hline Age & 0.038 & 0.017 & 5.069 & 1.039 & $1.005 \sim 1.074$ & 0.024 \\
tPSA & 0.025 & 0.008 & 9.853 & 1.026 & $1.010 \sim 1.042$ & 0.002 \\
fPSA & 0.195 & 0.048 & 16.28 & 1.215 & $1.106 \sim 1.336$ & $<0.001$ \\
PV & -0.028 & 0.006 & 22.599 & 0.972 & $0.961 \sim 0.983$ & $<0.001$ \\
DRE & 1.158 & 0.292 & 15.782 & 3.185 & $1.798 \sim 5.641$ & $<0.001$ \\
TRUS & 1.517 & 0.254 & 35.734 & 4.56 & $2.773 \sim 7.500$ & $<0.001$ \\
BMI & 0.617 & 0.166 & 13.714 & 1.852 & $1.337 \sim 2.567$ & $<0.001$ \\
Constant & -4.934 & 1.312 & 14.15 & 0.007 & & $<0.001$ \\
\hline
\end{tabular}

2 


\section{Table 4 (on next page)}

Diagnostic values of model and clinical parameters in the development group and validation group for the results of prostate biopsy

Abbreviations: dev, development group; val, validation group; tPSA, total prostate-specific antigen; \%fPSA, the ratio of fPSA to tPSA; PSAD, prostate-specific antigen density; SEN, sensitivity; SPE, spevificity; PPV, positive predictive value; NPV, negative predictive value; FNR, false negative rate; FPR, false positive rate; $A U C$, area under the curve; $\mathrm{Cl}$, confidence interval 


\begin{tabular}{cccccccccccc}
\hline Variable & cutoff & Youden index & $\begin{array}{c}\text { SEN } \\
(\%)\end{array}$ & $\begin{array}{c}\text { SPE } \\
(\%)\end{array}$ & $\begin{array}{c}\text { PPV } \\
(\%)\end{array}$ & $\begin{array}{c}\text { NPV } \\
(\%)\end{array}$ & $\begin{array}{c}\text { FNR } \\
(\%)\end{array}$ & $\begin{array}{c}\text { FPR } \\
(\%)\end{array}$ & AUC & $\begin{array}{c}95 \% \text { CI } \\
\begin{array}{c}P \text {-value } \\
\text { (AUC) }\end{array}\end{array}$ \\
\hline Model(dev) & $>0.31$ & 0.59 & 73 & 85.8 & 65.5 & 89.6 & 27 & 14.2 & 0.856 & $0.824 \sim 0.885$ & $<0.001$ \\
tPSA & $>31.5$ & 0.36 & 49.3 & 86.5 & 57.5 & 82.2 & 50.7 & 13.5 & 0.713 & $0.673 \sim 0.751$ & $<0.001$ \\
\%PSA & $<0.17$ & 0.28 & 46.6 & 81.5 & 48.3 & 80.5 & 53.4 & 18.5 & 0.624 & $0.582 \sim 0.665$ & $<0.001$ \\
PSAD & $>0.44$ & 0.46 & 65.5 & 80 & 54.8 & 86.3 & 34.5 & 20 & 0.761 & $0.723 \sim 0.797$ & $<0.001$ \\
Model(val) & $>0.31$ & 0.49 & 62.2 & 87.5 & 68.3 & 84.3 & 37.8 & 12.5 & 0.797 & $0.724 \sim 0.859$ & $<0.001$ \\
tPSA & $>31.5$ & 0.24 & 44.4 & 80 & 48.8 & 76.9 & 55.6 & 20 & 0.662 & $0.580 \sim 0.737$ & $<0.001$ \\
\%fPSA & $<0.17$ & 0.26 & 53.3 & 73.1 & 46.2 & 78.4 & 46.7 & 26.9 & 0.624 & $0.541 \sim 0.702$ & $<0.001$ \\
PSAD & $>0.44$ & 0.31 & 53.3 & 77.9 & 51.1 & 79.4 & 46.7 & 22.1 & 0.673 & $0.592 \sim 0.748$ & $<0.001$ \\
\hline
\end{tabular}




\section{Table 5 (on next page)}

Net benefit and reduction of the prediction model and other variables in the development and validation group

Abbreviations: dev, development group; val, validation group. 


\begin{tabular}{ccccc}
\hline Threshold & & & & \\
probability(\%) & dev & 31 & val & 31 \\
\hline & Model & 15.1 & Model & 13.9 \\
& tPSA & 7.9 & tPSA & 6.5 \\
Net benefit(\%) & \%PSA & 4.6 & \%PSA & 5.5 \\
& PSAD & 10.3 & PSAD & 8.6 \\
& Treat all & -5.8 & Treat all & -1.2 \\
& & & & \\
& Model & 46.5 & Model & 33.5 \\
& tPSA & 30.5 & tPSA & 17 \\
& \%fPSA & 28.5 & \%PSA & 14.9 \\
& PSAD & 35.8 & PSAD & 21.7 \\
\hline
\end{tabular}

2 


\section{Table 6(on next page)}

Diagnostic accuracy of our model and other models using validation group

Abbreviations: AUC, area under the curve; $\mathrm{Cl}$, confidence interval; val, validation group (149 cases); val*, validation group (136 cases) 
1

\begin{tabular}{cccc}
\hline Prediction model & AUC & $95 \%$ CI & $P$-value (AUC) \\
\hline Our model (val) & 0.797 & $0.724 \sim 0.859$ & $<0.001$ \\
Domestic Model 1 & 0.739 & $0.661 \sim 0.808$ & $<0.001$ \\
Domestic Model 2 & 0.753 & $0.676 \sim 0.820$ & $<0.001$ \\
Domestic Model 3 & 0.694 & $0.613 \sim 0.766$ & $<0.001$ \\
\hline Our model (val $^{*}$ ) & 0.793 & $0.715 \sim 0.857$ & $<0.001$ \\
PCPT model & 0.668 & $0.582 \sim 0.746$ & $<0.001$ \\
\hline
\end{tabular}




\section{Table 7 (on next page)}

Comparison of diagnostic values of other prediction models with that of our model

Abbreviations: AUC, area under the curve; val, validation group (149 cases); val*, validation group (136 cases) 


\begin{tabular}{ccc}
\hline Prediction model & AUC & $P$-value \\
\hline Our model (val) & 0.797 & N/A \\
Domestic Model 1 & 0.739 & 0.1471 \\
Domestic Model 2 & 0.753 & 0.2424 \\
Domestic Model 3 & 0.694 & 0.0148 \\
\hline Our model (val*) $^{*}$ PCPT-CRC & 0.793 & $\mathrm{~N} / \mathrm{A}$ \\
PCPT & 0.668 & 0.032 \\
\hline
\end{tabular}

1 